\title{
COMPARISON OF MACROMOLECULAR COMPONENT DISTRIBUTIONS IN OSTEOARTHRITIC AND HEALTHY CARTILAGES BY FOURIER TRANSFORM INFRARED IMAGING
}

\author{
JIANHUA YIN*, ${ }^{*}$, YANG XIA ${ }^{\dagger}$ and ZHIYAN XIAO* \\ *Department of Biomedical Engineering \\ Nanjing University of Aeronautics and Astronautics \\ Nanjing 210016, P. R. China \\ ${ }^{\dagger}$ Department of Physics and Center for Biomedical Research \\ Oakland University, Rochester, MI 48309, USA \\ ¿Department of Radiation Oncology \\ Washington University School of Medicine \\ St. Louis, MO 63110, USA \\ §yin@nuaa.edu.cn
}

Received 4 May 2013

Accepted 19 August 2013

Published 4 October 2013

\begin{abstract}
Fourier transform infrared imaging (FTIRI) was used to examine the depth-dependent content variations of macromolecular components, collagen and proteoglycan (PG), in osteoarthritic and healthy cartilages. Dried $6 \mu \mathrm{m}$ thick sections of canine knee cartilages were imaged at $6.25 \mu \mathrm{m}$ pixel-size in FTIRI. By analyzing the infrared (IR) images and spectra, the depth dependence of characteristic band (sugar) intensity of PG show obvious difference between the cartilage sections of $(\mathrm{OA})$ and health. The result confirms that PG content decreases in the osteoarthritic cartilage. However, no clear change occurs to collagen, suggesting that the OA influences little on the collagen content at early stage of OA. This observation will be helpful to further understand PG loss associated with pathological conditions in OA, and demonstrates that FTIRI has the potential to become an important analytical tool to identify early clinical signs of tissue degradation, such as PG loss even collagen disruption.
\end{abstract}

Keywords: Macromolecular component; osteoarthritis; articular cartilage; Fourier transform infrared imaging (FTIRI).

$\S$ Corresponding author.

This is an Open Access article published by World Scientific Publishing Company. It is distributed under the terms of the Creative Commons Attribution 3.0 (CC-BY) License. Further distribution of this work is permitted, provided the original work is properly cited. 


\section{Introduction}

Articular cartilage $(\mathrm{AC})$ is the connective tissue that covers the articulating surfaces of bones in synovial joints to provide a nearly frictionless surface for normal joint motion and to withstand external loading. AC has two principal macromolecular components (type II collagen and proteoglycan $(\mathrm{PG})$ ) and is saturated with water and mobile ions, ${ }^{1-3}$ which compose extracellular matrix (ECM). The collagen fibers are woven into a threedimensional fibros network, which enmeshes PG molecules in the tissue.

Based on a depth-dependent orientation the fibrous network in ECM, uncalcified cartilage can be subdivided into three structural zones, that is a superficial zone (SZ), a transitional zone (TZ), and a radial zone (RZ) from the surface to subchondral bone, ${ }^{1-5}$ respectively. The zonal difference in anisotropy, distribution of components, shape, and arrangement of chondrocytes ${ }^{2}$ provides AC with the functional properties of depth-dependent tensile strength and resiliency. ${ }^{4,6,7}$ However, once there are characteristic changes in reduction in the PG content, alteration in collagen fibril structure, and increased synthesis and degradation of matrix macromolecules ${ }^{8}$ in cartilage, they would signal the onset of functional degeneration of the tissue, which will eventually lead to degenerative joint diseases such as osteoarthritis (OA) or rheumatoid arthritis. ${ }^{1}$

Fourier transform infrared spectroscopic imaging (FTIRI) is a newly developed vibrational spectroscopy technique with fine spatial and spectral resolutions. ${ }^{9-17}$ FTIRI is sensitive to the chemical composition of bio-tissue and can be used to investigate tissue material properties and their spatial distribution, ${ }^{18}$ such as the collagen and $\mathrm{PG}$ content distribution with microscopic resolution. ${ }^{9,10}$

Some factors, such as an acute anterior cruciate ligament (ACL) injury and aging, would result in chondral lesions or OA. Therefore, the purpose of the current FTIRI study was to detect the macromolecular component changes in cartilage, where OA was induced by the ACL-transection. We addressed the hypothesis that PG content decreases at SZ and no change happens for collagen by comparing with the control specimen. Cartilage quality was also assessed by FTIRI spatially resolved analysis of chondrocytes.

\section{Materials and Methods}

\subsection{Sample preparation}

Eighteen intact knees were harvested from nine 2year old dogs after they were sacrificed. They include four healthy dogs, five injured dogs that had been transected for eight-week on ACL in one knee joint and had similar individual features in age and weight. Articular cartilage attaching on bones were cut from the joints by using a table saw and a diamond blade to get some blocks. $10-\mu \mathrm{m}$ thick sections were cut from each frozen block in cryostat (Leica CM 1950, Germany) and mounted on MirrIR slides (Kevley Technologies, Chesterland, $\mathrm{OH}$ ).

\subsection{FTIR imaging}

After air dry for $2 \mathrm{~h}$, each cartilage section was imaged using a Perkin Elmer Spotlight 300 Infrared Imaging system (Wellesley, MA) at $6.25-\mu \mathrm{m}$ pixel resolution and $16 \mathrm{~cm}^{-1}$ spectral resolution. The system is composed of an Fourier transform infrared (FTIR) spectrometer, an IR microscope, and a liquid $\mathrm{N}_{2}$ cooled 16-element (mercuric cadmium telluride) MCT array detector and a single point MCT detector. An internal coaxial LED illumination with variable intensity is available in the instrument to produce visible images, which enables the identification of the tissue region for IR imaging.

In the reflection imaging mode, the infrared light focused on the specimen that is mounted on a movable mechanical stage. The data were collected by the array detector. Background (MirrIR slide) spectra were collected for each section analyzed, and were used for correction of the sample spectral data over a range of $4000-744 \mathrm{~cm}^{-1}$.

Most FTIRI studies of articular cartilage have used the peak intensities/areas in the infrared absorption images to represent the molecular contents. ${ }^{14-19}$ During the image analysis, the characteristic band intensity images (chemimaps) can be obtained by fixing the spectral range of the corresponding characteristic bands, including Amide I $\left(1700-1600 \mathrm{~cm}^{-1}\right)$, amide II $\left(1600-1500 \mathrm{~cm}^{-1}\right)$, amide III $\left(1300-1200 \mathrm{~cm}^{-1}\right)$ and sugar $\left(1125-960 \mathrm{~cm}^{-1}\right)$ bands.

The characteristic absorbance (absorption intensity) of each column would be calculated by averaging the all spectra from each pixel within the same column in FTIR image. The average absorbance with standard deviation along the direction of 
the cartilage depth is the depth profile of absorbance or component.

\subsection{Statistical analysis}

Statistical analysis was performed by using commercial software Kaleida-Graph (v4.0, Synergy Software, Reading, PA). PG data of both groups of the healthy and osteoarthritic sections were compared with each other using the non-parametric Wilcoxon-Mann-Whitney test. In WilcoxonMann-Whitney test, if the $p$-value is below 0.05 , the conclusion is that there is a difference between both groups.

\section{Results}

Figure 1 shows an IR spectrum extracted from the FTIR images of a healthy cartilage by the Spotlight software of PerkinElmer. Two characteristic peaks of collagen were assigned to amides I and II, respectively. ${ }^{11-13}$ The sugar band centering at $1072 \mathrm{~cm}^{-1}$ was attributed to PG. ${ }^{11-13}$ The characteristic bands play significant roles in structural changes and macromolecular component discrimination. Therefore, based on the IR band assignments, the corresponding FTIR images of the signature bands for the healthy cartilage section were obtained by the Spotlight software and shown in Fig. 2. It exhibits in turn the visible image, total absorption image, amides I, II, III images and

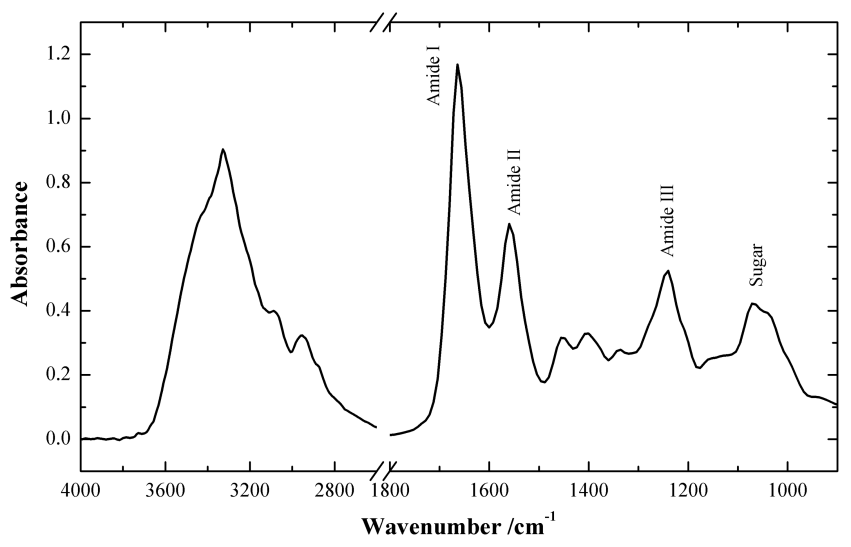

Fig. 1. An IR spectrum from the FTIRI image of the healthy articular cartilage. Two characteristic peaks of collagen were assigned to amides I and II band, and sugar $\left(1072 \mathrm{~cm}^{-1}\right)$ band was attributed to PG. The spectral ranges for amides I, II, III and sugar are $1700-1600 \mathrm{~cm}^{-1}, 1600-1500 \mathrm{~cm}^{-1}, 1300-$ $1200 \mathrm{~cm}^{-1}$, and $1125-960 \mathrm{~cm}^{-1}$, respectively.
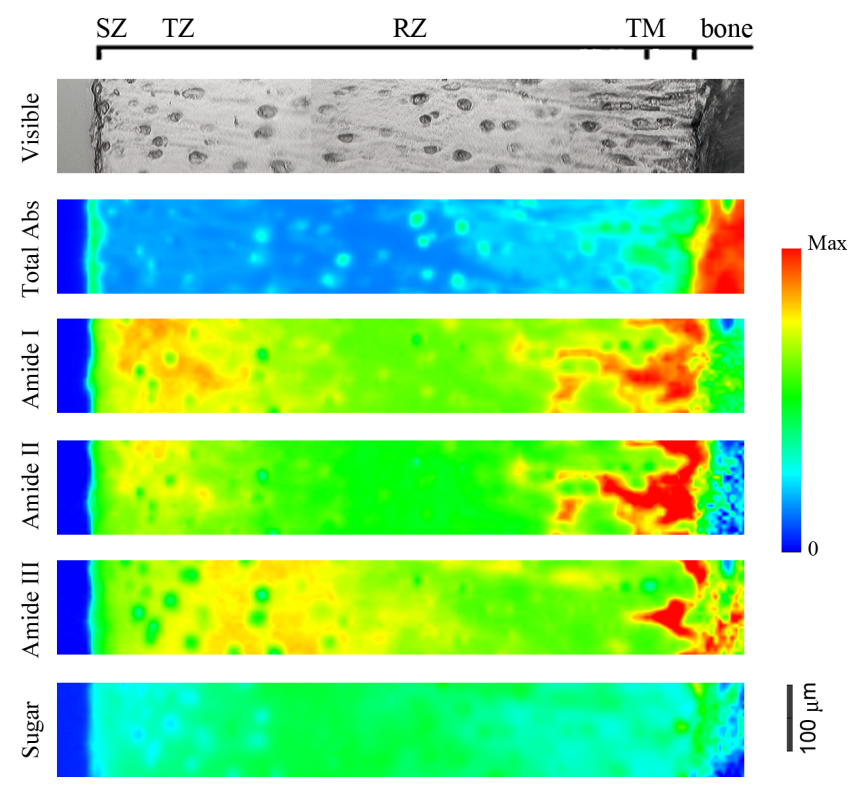

Fig. 2. A set of images of a typically healthy cartilage section, from the top to the bottom, including visible image, total IR absorption image, amides I, II, III images, and $1072 \mathrm{~cm}^{-1}$ sugar image, respectively. The same color scale was used to plot the FTIRIs, with the max absorptions for the images from the top to the bottom being $1.45,0.5,0.35,0.15$, and 0.30 , respectively. The black bar presents 100 microns. The division of the histological zones was based on the previous studies ${ }^{14,16}$ : SZ: superficial zone; TZ: transitional zone; RZ: radial zone; TM: tidemark.

$1072 \mathrm{~cm}^{-1}$ band image from the top to the bottom. One can find that the absorption values in these FTIR images are rather inhomogeneous, represented by the artificial colors in the images. Therefore, the spectroscopic band images of amide I (II) and sugar bands represent qualitatively the inhomogeneous distributions of collagen and $\mathrm{PG}$ across the uncalcified tissue (SZ, TZ, and RZ), respectively.

Similarly, a set of the FTIR images of the signature bands for the osteoarthritic cartilage section obtained from an eight-week ACL-transected knee is shown in Fig. 3. It also includes the corresponding visible image, total absorption image, amides I and II images (chemimaps of collagen), sugar band image (chemimap of $\mathrm{PG}$ ) of the tissue section, respectively. A common result was obtained in Figs. 2 and 3 that the PG has a relative lower content at TZ by comparing to that at the SZ and the second half of RZ under the dry weight condition.

The variations of collagen and PG contents with cartilage depth in the healthy and the OA cartilage sections can be observed by making chemimaps and 


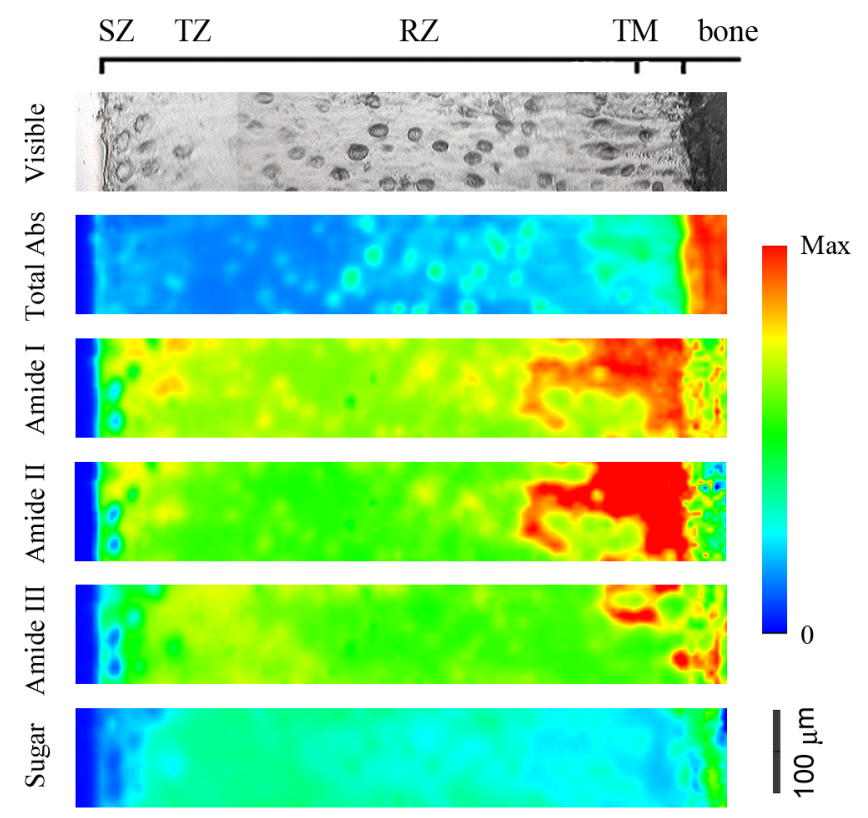

Fig. 3. A set of images of an typically induced osteoarthritic cartilage section from the top to the bottom, in turn visible image, total IR absorption image, amides I, II, III images, and $1072 \mathrm{~cm}^{-1}$ sugar image. The same color scale was used to plot the FT-IR images, with the max absorptions for the images from the top to the bottom being $1.45,0.5,0.35,0.15$, and 0.30 , respectively. The black bar presents 100 microns.

extracting the absorption values of region of interest. Figure 4 shows the depth-dependent profiles of the amides I, II, III and sugar $\left(1072 \mathrm{~cm}^{-1}\right)$ absorption bands through the cartilage depth from the corresponding chemimaps. The intuitive profiles of the characteristic absorption values across the uncalcified cartilage were compared between the healthy and the osteoarthritic section. For the osteoarthritic section, it is relevant firstly to note that the absorbance profile of amide I band with depth is very close to that of the health, as well as the depth dependent profile of amide II. The consistencies in Fig. 4(a) or 4(b) reveal that the collagen content and distribution across the uncalcified cartilage are in agreement between the healthy and the osteoarthritic sections. On the other hand, the absorbance profile of amide I band with depth is very similar to that of amide II band either for the healthy section or the osteoarthritic section, as shown in Figs. 4(a) and 4(b). The results suggest that it is effective to mainly assign the amides I and II to the type II collagen in cartilage.

Figure 4(c) shows the absorbance profiles of characteristic absorption band of $1072 \mathrm{~cm}^{-1}$ (sugar) across the uncalcified cartilage of the healthy and the osteoarthritic sections, respectively. Both profiles display clear difference in the absorption value. At the SZ, the absorption values of the osteoarthritic section are obviously lower than those of the healthy one, indicating that the $\mathrm{PG}$ content decreases at the zone due to the eight-week ACLtransection. The statistical analysis was completed for the comparison of the PG content of the osteoarthritic group with that of the healthy group at the SZ using the non-parametric WilcoxonMann-Whitney test. The $p$-value $(p<0.0001)$ suggests that the PG value of osteoarthritic group were significantly different from the healthy group. The collagen content of osteoarthritic group by means of amide II were not significant different with that of the healthy group $(p=0.22)$. In the range of 0.095-0.285 (TZ), the PG content are in agreement with each other.

The depth-dependent profiles of the amide III band for both kinds of cartilage sections are displayed in Fig. 4(d). No matter in the healthy section or the osteoarthritic section, amide III absorbance profiles differs to the corresponding profile of amide I or amide II band. But there are some similarities at different partition. For example, both profiles show proximity in the range from 0.1 to 0.3 . The results above might be ascribed that the amide III band is a complex band of the collagen and PG with the variational content contributions at different regions.

\section{Discussion}

The corresponding FTIRIs and the profiles of absorbance qualitatively represent the distributions of the macromolecular components (collagen and $\mathrm{PG}$ ) in the cartilage sections. For the collagen content distribution, no clear change is shown in the absorbance profiles of collagen bands, suggesting that the OA influences little on the collagen content at early stage of OA. For the osteoarthritic section, the lower absorption values of $1072 \mathrm{~cm}^{-1}$ band at the SZ than those of the healthy one reveals that the PG content decreases even if undergoing early OA duration of eight weeks. Meanwhile, the absorbance decreases again after $\mathrm{TZ}$, relative to those of healthy section. Therefore, the induced OA disease (shown as PG content change) might affect the PG content distribution at the RZ.

Additionally, the embedded chondrocytes at the $\mathrm{SZ}$ also become blue, when the remarkable bluing 


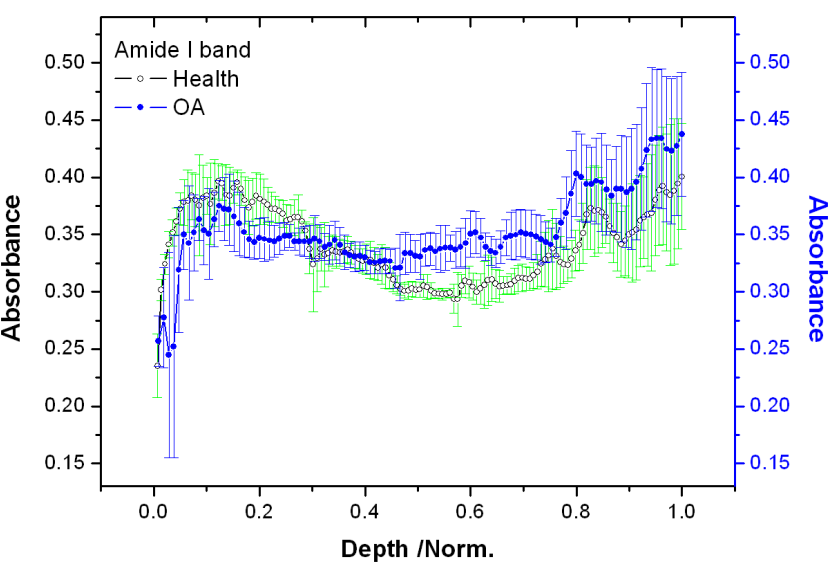

(a)

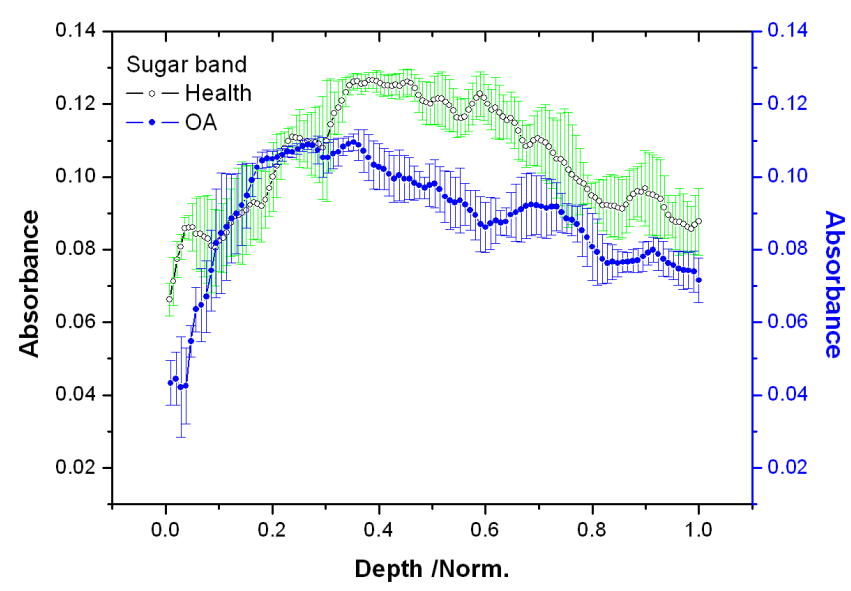

(c)

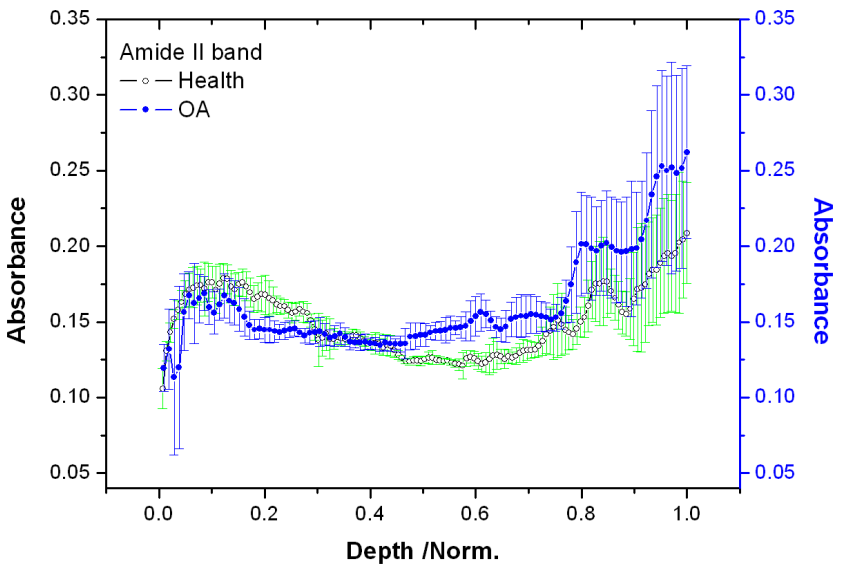

(b)

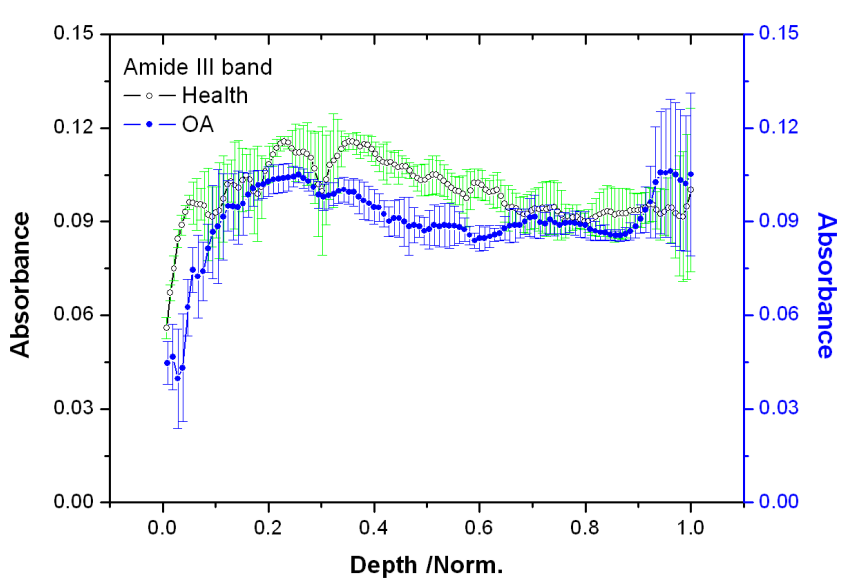

(d)

Fig. 4. The comparisons of the absorption intensity profiles of (a) amide I, (b) amide II, (c) sugar and (d) amide III across the entire depths of the healthy and osteoarthritic cartilage sections. The absorption values are represented by using the hollow and solid circles for the healthy and osteoarthritic samples, respectively. The error bar implies the standard deviation of the absorbance.

appears at the SZ (0-0.1) in the sugar chemimap (in Fig. 3). It suggests that the absorption and scattering of chondrocytes become obviously week and consequently might reveal that the contents in chondrocytes and peripheral ECM altered, even the functional activity of chondrocytes to synthesize PG changed. ${ }^{20}$ This claim can be further confirmed by the deformation of the chondrocytes at the SZ. As shown in the visible images in Figs. 2 and 3, the shape of chondrocytes at the SZ changed from the small ellipse under normal condition (Fig. 2) to the bigger circle in OA disease (Fig. 3). These results demonstrate that the FTIRI can be used as a helpful tool to monitor the variations in component, functional activity, and disease in bio-tissues.

Both collagen and PG have their own characteristic bands in FTIR spectra, respectively, ${ }^{14-19}$ as mentioned above. Actually, there is some overlapping between the spectra of collagen and PG in the interested wavenumber range; consequently, a direct interpretation of characteristic band images/absorbance profiles as the molecular concentration distributions will contain error. Hence, these spectral band intensity profiles ${ }^{14-19}$ can only be used for qualitative or semiquantitative analysis of molecular content, not accurate interpretation of the molecular concentration. In order to accurately quantify the collagen and $\mathrm{PG}$ concentrations, it is necessary to combine FTIRI with chemometrics algorithms in future. However, if the need is for qualitative and semiquantitative analysis without the accurate requirement in molecular concentration, FTIRI tool has significant sensitivity to be competent to the related researches in biology and biomedicine. 


\section{Conclusion}

FTIRI technique was used to study the variations in chemical compositions in the healthy and osteoarthritic cartilages. The comparisons of the depth dependent profiles of characteristic band intensity disclose that the collagen content just displays slight fluctuations between the healthy cartilage and the osteoarthritic cartilage eight-week after the ACL-transection. However, the PG content clearly decreases in the osteoarthritic cartilage accompanying with the deformation of chondrocytes at the SZ of the osteoarthritic cartilage. This observation will be helpful to monitor the $O A$ disease processes and to further understand PG loss associated with osteoarthritic injury and repair by comparing the PG loss in different conditions. Additionally, this chemical imaging approach with fine spatial and spectral resolutions has the potential to become an important analytical tool to quantitatively analyze and identify early clinical signs of tissue degradation, such as PG loss even collagen disruption.

\section{Acknowledgments}

The authors are grateful to the National Institutes of Health in U.S.A. for the R01 grants (AR 045172, AR 052353) to Yang Xia. The authors are indebted to Dr. John Matyas (Veterinary Medicine, University of Calgary, Canada) for providing the lesioned specimens to an ongoing project, Drs. Clifford Les and Hani Sabbah (Henry Ford Hospital, Detroit) for providing the healthy specimens, and the students and staff of the Xia Lab at Oakland University (Dr. Nian Wang, Mr. Farid Badar, Mr. Daniel Mittelstaedt, Ms Jihyun Lee) for preparing the cartilage specimen blocks.

\section{References}

1. J. C. Y. Hu, K. A. Athanasiou, "Structure and function of articular cartilage," Handbook of Histology Methods for Bone and Cartilage, Y. H. An, K. L. Martin, Eds., pp. 73-95, Humana Press, Totowa, New Jersey, (2003).

2. V. C. Mow, W. Y. Gu, F. H. Chen, "Structure and function of articular cartilage and meniscus," Basic Orthopaedic Biomechanics and Mechano-Biology, R. H. Van, C. Mow, Eds., pp. 181-258, Lippincott Williams \& Wilkins, Philadelphia, (2005).
3. V. C. Mow, X. E. Guo, "Mechano-electrochemical properties of articular cartilage: Their inhomogeneities and anisotropies," Ann. Rev. Biomed. Eng. 4, 175-209 (2002).

4. A. Maroudas, "Balance between swelling pressure and collagen tension in normal and degenerate cartilage," Nature 260, 808-809 (1976).

5. J. A. Buckwalter, H. J. Mankin, "Articular cartilage.1. Tissue design and chondrocyte-matrix interactions," J. Bone Joint Surg. Am. 79A, 600-611 (1997).

6. S. S. Chen, Y. H. Falcovitz, R. Schneiderman, A. Maroudas, R. L. Sah, "Depth-dependent compressive properties of normal aged human femoral head articular cartilage: Relationship to FCD," Osteoarthritis and Cartilage 9, 561-569 (2001).

7. W. Wilson, J. M. Huyghe, C. C. V. Donkelaar, "Depth-dependent compressive equilibrium properties of articular cartilage explained by its composition," Biomech. Model Mechanobiol. 6, 4353 (2007).

8. K. D. Brandt, H. J. Mankin, L. E. Shulman, "Workshop on etiopathogenesis of osteoarthritis," J. Rheumatol 13, 1126-1160 (1986).

9. J. H. Yin, Y. Xia, "Macromolecular concentrations in bovine nasal cartilage by Fourier transform infrared imaging and principal component regression," Appl. Spectrosc. 64, 1199-1208 (2010).

10. J. H. Yin, Y. Xia, "Chemical visualization of individual chondrocytes in articular cartilage by attenuated-total-reflection Fourier transform infrared microimaging," Biomed. Opt. Express 2, 937-945 (2011).

11. Y. Xia, N. Ramakrishnan, A. Bidthanapally, "The depth-dependent anisotropy of articular cartilage by Fourier-transform infrared imaging (FTIRI)," Osteoarthritis and Cartilage 15, 780-788 (2007).

12. K. Potter, L. H. Kidder, I. W. Levin, E. N. Lewis, R. G. Spencer, "Imaging of collagen and proteoglycan in cartilage sections using Fourier transform infrared spectral imaging," Arthritis Rheum. 44, 846-855 (2001).

13. N. P. Camacho, P. West, P. A. Torzilli, R. Mendelsohn, "FTIR microscopic imaging of collagen and proteoglycan in bovine cartilage," Biopolymers 62, 1-8 (2001).

14. J. H. Lee, Y. Xia, Quantitative zonal differentiation of articular cartilage by microscopic magnetic resonance imaging, polarized light microscopy, and Fourier-transform infrared imaging, Microsci. Res. Tech. 76, 625-632 (2013).

15. X. Bi, X. Yang, M. P. G. Bostrom, D. Bartusik, S. Ramaswamy, K. W. Fishbein, R. G. Spencer, N. P. Camacho, "Fourier transform infrared imaging and MR microscopy studies detect compositional and 
structural changes in cartilage in a rabbit model of osteoarthritis," Anal. Bioanal. Chem. 387, 16011612 (2007).

16. N. Ramakrishnan, Y. Xia, A. Bidthanapally, M. Lu, "Determination of zonal boundaries in articular cartilage using infrared dichroism," Appl. Spectrosc. 61, 1404-1409 (2007).

17. Y. Xia, H. Alhadlaq, N. Ramakrishnan, A. Bidthanapally, F. Badar, M. Lu, "Molecular and morphological adaptations in compressed articular cartilage by polarized light microscopy and Fourier-transform infrared imaging," J. Struct. Biol. 164, 88-95 (2008).

18. Ericka F. Calton, Jennifer MacLeay, Adele L. Boskey, "Fourier transform infrared imaging analysis of cancellous bone in alendronateand raloxifene-treated osteopenic sheep," Cells Tissues Organs 194, 302-306 (2011).

19. X. Bi, X. Yang, M. P. Bostrom, N. P. Camacho, "Fourier transform infrared imaging spectroscopy investigations in the pathogenesis and repair of cartilage," Biochim. Biophys. Acta 1758, 934-941 (2006).

20. J. A. Ryan, E. A. Eisner, G. DuRaine, Z. You, A. Hari Reddi, "Mechanical compression of articular cartilage induces chondrocyte proliferation and inhibits proteoglycan synthesis by activation of the ERK pathway: Implications for tissue engineering and regenerative medicine," J. Tissue Eng. Regen. Med. 3, 107-116 (2009). 